\title{
Zahnfarbene Werkstoffe für festsitzenden Zahnersatz und ihre Funktion - was wissen wir?
}

Anuschka Josephine Roesner, Andreas König, Elena Günther, Sebastian Hahnel, Angelika Rauch

\author{
Dank der kontinuierlichen Weiterentwicklung im Bereich der zahnärztlichen Mate- \\ rialien stehen uns heute verschiedenste zahnfarbene Werkstoffe zur Verfügung, \\ die dem Wunsch nach ästhetischen und biokompatiblen festsitzenden zahnärzt- \\ lichen Versorgungen in besonderem Maße gerecht werden. Mit dem vorliegenden \\ Artikel sollen die verschiedenen, sich gegenwärtig auf dem Markt befindenden \\ zahnfarbenen Werkstoffe genauer beleuchtet, ihre Indikationsfelder aufgezeigt \\ sowie ein Ausblick auf Möglichkeiten und Grenzen dargelegt werden.
}

\section{Zahnfarbene Werkstoffe - welche gibt es?}

Bei zahnfarbenen Werkstoffen handelt es sich um eine Sammelbezeichnung, unter der zunächst Dentalkeramiken und Kunststoffe (Polymere) zusammengefasst werden können. Abhängig von ihrem jeweiligen Einsatzgebiet können sie als Gerüstmaterialien, als Verblendwerkstoffe oder zur monolithischen Anwendung geeignet sein.

Zahnfarbene Werkstoffe können aber auch anhand ihres Herstellungsprozesses differenziert werden. So können Restaurationen aus keramischen Werkstoffen entweder im sogenannten Lost-Wax-Verfahren gepresst, computergestützt konstruiert und gefräst (CAD/CAM für Computer-Aided-Design/Computer-Aided-Manufacturing)

[1,2] oder seit Kurzem auch additiv im 3-D-Druckverfahren $[3,4]$ gefertigt werden. Für eine keramische Verblendung kann die Schicht-/Schlickertechnik angewendet werden. Dabei wird im konventionellen Verfahren durch Anmischen von Pulver und Flüssigkeit die Verblendmasse mehrfach geformt und kondensiert, bevor sie dann unter Vakuum gebrannt wird. Mittlerweile können Verblendungen auch im digitalen Verfahren hergestellt werden (z. B. CAD-on-Technik). Restaurationen aus Kompositen können additiv oder subtraktiv in einem CAD/CAM-Prozess hergestellt werden. Bei den digitalen Verfahren erfolgt direkt am Behandlungsstuhl eine Abformung mittels Intraoralscanner oder nach konventioneller Abformung eine Digitalisierung der Modelle durch einen Laborscanner. Danach werden die Restaurationen mithilfe einer CAD-Software konstruiert, um den Datensatz anschließend an eine Fräseinheit oder einen Drucker zu übermitteln, worin die Restauration schließlich gefertigt wird.
Eine weitere Möglichkeit der Differenzierung zahnfarbener Werkstoffe kann aufgrund der Zusammensetzung erfolgen ( $\triangleright$ Abb. 1). So können die Dentalkeramiken in Silikatkeramiken, Oxidkeramiken sowie polymerinfiltrierte Keramiken (PICN) unterteilt werden. Letztere bestehen aus 2 miteinander verbundenen netzartigen Strukturen, einem Polymer- und einem Feldspatkeramik-Netzwerk, weshalb polymerinfiltrierte Keramiken nicht gebrannt werden dürfen. Aufgrund der Tatsache, dass bei PICN ein keramisches Feldspat-Netzwerk mit einem prozentualen Gewichtsanteil von $86 \%$ vorliegt und dieses im Vorfeld der intraoralen Befestigung der Restauration einer Vorbehandlung mit Flusssäure bedarf, wird die Gruppe der PICN in der vorliegenden Arbeit den dentalen Keramiken zugeordnet.

Darüber hinaus unterscheiden sich die jeweiligen zahnfarbenen Werkstoffe hinsichtlich ihrer spezifischen mechanischen Eigenschaften. So sind für die Bewertung des Bruchverhaltens 2 mechanische Eigenschaften von besonderem Interesse. Die Biegefestigkeit gibt die maximale Spannung (Kraft bezogen auf eine Bezugsfläche) an, die der Werkstoff aufnehmen kann. Je größer die Biegefestigkeit ist, umso höhere Kräfte können auf den Werkstoff einwirken, ohne dass dieser bricht. Diese Eigenschaft wird durch Biegeversuche ermittelt, wobei zwischen einaxialen, 3-Punkt- und 4-Punkt-Biegeversuchen an Biegebalken und biaxialen Biegeversuchen an Plättchen unterschieden werden muss, da die Ergebnisse abhängig vom angewandten Versuchsaufbau bzw. der Norm differieren. Die 2. relevante mechanische Werkstoffeigenschaft ist der Elastizitätsmodul (E-Modul). Der E-Modul beschreibt die Steifigkeit bzw. die lastabhängige Verformbarkeit eines Werkstoffes. 


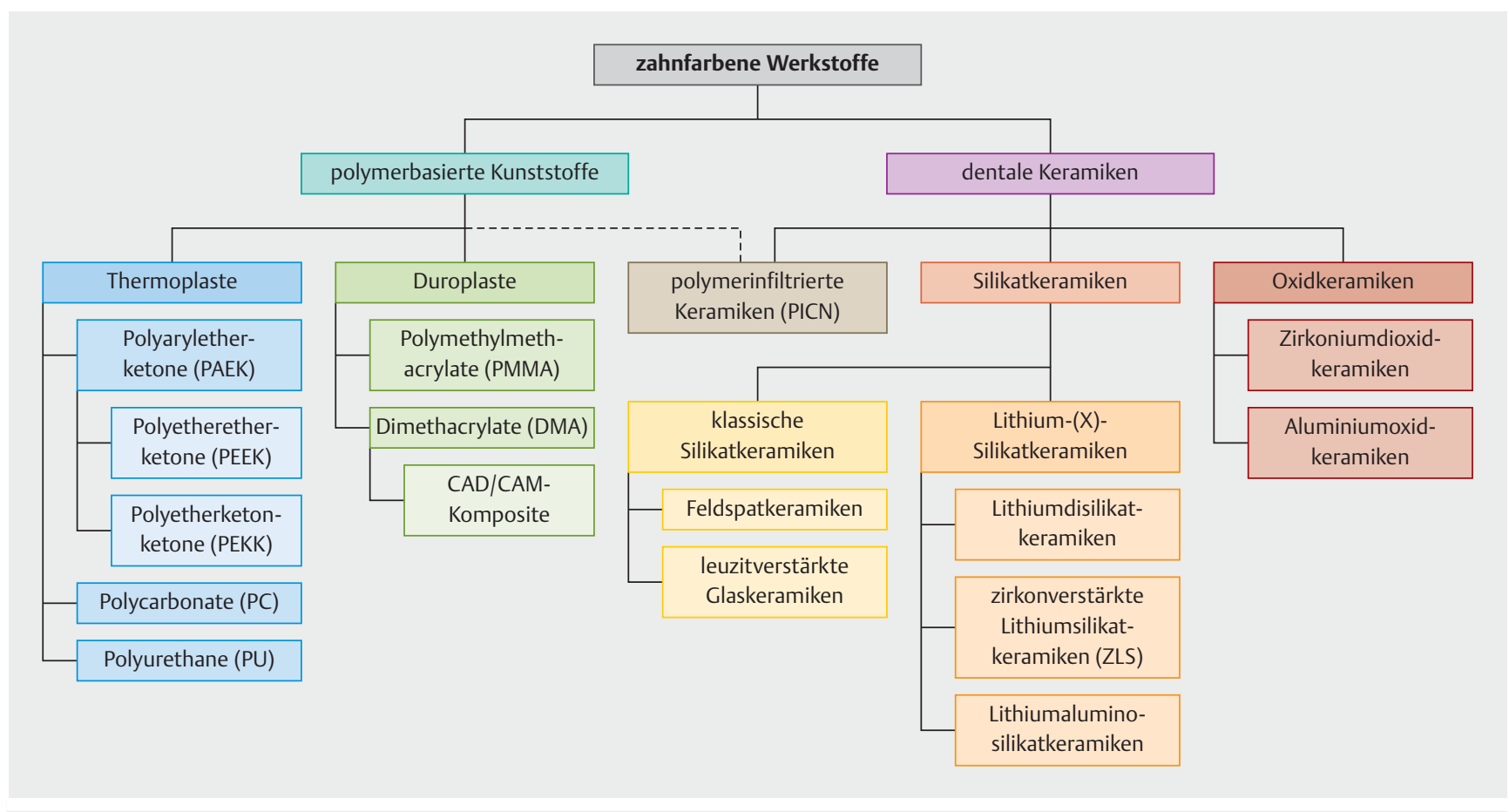

- Abb. 1 Schematische Darstellung zahnfarbener Werkstoffe.

Beide Eigenschaften sind wichtige Kriterien für die Festlegung der jeweiligen materialabhängigen Indikationsgebiete und bestimmen maßgeblich die für Restaurationen aus den jeweiligen Materialien zur Verfügung stehenden Befestigungsmöglichkeiten. In $>$ Tab. 1 sind die verschiedenen zahnfarbenen Werkstoffe und ihre materialspezifischen Eigenschaften aufgeführt und in > Tab. 2 ihre Indikationsspektren dargestellt.

\section{Kunststoffe (Polymere)}

Kunststoffe (Polymere) sind bereits seit Jahrzehnten in der Zahnmedizin als Füllungsmaterialien oder Werkstoffe zur Herstellung von Schienen oder Prothesen im Einsatz.

Bei den zahnfarbenen Kunststoffen, die für festsitzenden Zahnersatz verfügbar sind, können zunächst Thermoplaste und Duroplaste voneinander unterschieden werden. Zu der Gruppe der Thermoplaste zählen hierbei die Materialien Polyurethan (PU), Polycarbonat (PC) sowie Polyaryletherketon (PAEK) [5]. Bei letzterem handelt es sich um Hochleistungspolymere, die als gefüllte Polyethyletherketone (PEEK) und Polyetherketonketone (PEKK) bspw. als elfenbeinfarbene Variante für die Herstellung von festsitzendem Zahnersatz zur Verfügung stehen (Pekkton ivory, Cendres+Métaux SA, Biel, Schweiz). Da bis zum jetzigen Zeitpunkt die verfügbare Farbpalette von PAEK-Werkstoffen eingeschränkt ist, wird häufig noch eine Verblendung notwendig. Hierin liegt die besondere Herausforderung dieser Werkstoffe, denn der häufig beschriebene Vorteil der hohen Biokompatibilität aufgrund der inerten Oberfläche bedingt gleichzeitig auch eine reaktionsträge Oberfläche, die einen sicheren sowie dauerhaften Verbund zu Verblendmaterialien erschwert $[6,7]$.

In der Gruppe der Duroplaste sind die Dimethacrylate zu nennen, die zusammen mit anorganischen Füllern einen wesentlichen Bestandteil der Komposite bilden. Moderne Füllungskomposite sind den klassischen Amalgammaterialien nicht nur ästhetisch überlegen, sondern stellen mittlerweile höchst leistungsstarke Verbundwerkstoffe mit vielfältigem Einsatzspektrum dar [8]. Indirekte Komposite (CAD/CAM-Komposite) haben prinzipiell ähnliche Bestandteile wie Füllungs- und Verblendkomposite. So handelt es sich hierbei um ein Polymer aus Dimethacrylaten wie bspw. Bisphenol-A-Glyzidyl-Methacrylat (BisGMA), Triethylenglycoldimethacrylat (TEGDMA) oder Urethandimethacrylat (UDMA). Im Rahmen des industriellen Fertigungsprozesses werden CAD/CAM-Komposite unter hohen Temperaturen und Druck erzeugt. Hieraus resultieren die verbesserten Materialeigenschaften wie eine gesteigerte Homogenität und ein reduzierter Restmonomergehalt im Vergleich zu chemisch- bzw. lichthärtenden Kompositmaterialien [9]. Zudem verfügen sie über einen dentinähnlichen E-Modul, wodurch sie ein natürliches Kaugefühl begünstigen und als zahnhartsubstanzschonend gegenüber Antagonisten gelten [10]. Neben ihrem ursprünglichen Indikationsspektrum als Inlays, Onlays und Kronen sind mittlerweile 2 Vertreter der CAD/CAM-Komposite vom Hersteller auch für 3-gliedrige Brückenrestaurationen freigegeben (LuxaCam Composite, DMG Chemisch-Pharmazeutische Fabrik 
- Tab. 1 Übersicht über verschiedene zahnfarbene Werkstoffe, die gesammelten Daten stammen aus verschiedenen Buch-Publikationsquellen sowie von Herstellerangaben.

\begin{tabular}{|c|c|c|c|c|c|c|c|c|c|}
\hline & $\begin{array}{l}\text { CAD/CAM- } \\
\text { Komposite }\end{array}$ & PICN & Feldspatkeramiken & $\begin{array}{l}\text { leuzitverstärkte } \\
\text { Glaskeramiken }\end{array}$ & \multicolumn{2}{|c|}{$\begin{array}{l}\text { Lithium-X-Silikat- } \\
\text { keramiken }\end{array}$} & \multicolumn{3}{|c|}{$\begin{array}{l}\text { Zirkoniumdioxid- } \\
\text { keramiken }\end{array}$} \\
\hline Beispiele & $\begin{array}{l}\text { Grandio blocs, } \\
\text { VOCO } \\
\text { LuxaCam } \\
\text { Composite, } \\
\text { DMG }\end{array}$ & $\begin{array}{l}\text { VITA ENAMIC, } \\
\text { VITA Zahn- } \\
\text { fabrik }\end{array}$ & $\begin{array}{l}\text { VITABLOCS Mark II, } \\
\text { VITA Zahnfabrik } \\
\text { VITA VM9 (Verblen- } \\
\text { dung), VITA Zahnfabrik }\end{array}$ & $\begin{array}{l}\text { IPS Empress CAD, } \\
\text { Ivoclar Vivadent }\end{array}$ & \multicolumn{2}{|c|}{$\begin{array}{l}\text { IPS e.max CAD, } \\
\text { Ivoclar Vivadent } \\
\text { Celtra Press, } \\
\text { Dentsply Sirona }\end{array}$} & \multicolumn{3}{|c|}{$\begin{array}{l}\text { monolithisch: Katana } \\
\text { UTML; Kuraray } \\
\text { Gerüst: LAVA Frame } \\
\text { Zirkonoxid, 3M }\end{array}$} \\
\hline $\begin{array}{l}\text { Biegefestigkeit } \\
\text { (MPa) }\end{array}$ & $100-250$ & 150 & $96-112$ & $160-180$ & \multicolumn{2}{|l|}{$350-600$} & \multicolumn{3}{|c|}{$\begin{array}{l}\text { 700-1500 (abhängig } \\
\text { von der Generation) }\end{array}$} \\
\hline E-Modul (GPa) & $10-20$ & 30 & 45 & 62 & \multicolumn{2}{|l|}{$70-95$} & \multicolumn{3}{|c|}{$\geq 200$} \\
\hline Härte (HV) & $1-2$ & 2,5 & $5-6$ & 6 & \multicolumn{2}{|l|}{$6-7$} & \multicolumn{3}{|l|}{12} \\
\hline $\begin{array}{l}\text { Erforderliche } \\
\text { Vorbehand- } \\
\text { lung }\end{array}$ & $\begin{array}{l}\text { Strahlen } \\
(50 \mu \mathrm{m} / 2 \text { bar })\end{array}$ & \multicolumn{3}{|c|}{ Ätzen mit Flusssäure (HF) für 60 Sek. } & \multicolumn{2}{|c|}{ Ätzen (HF) 20 Sek. } & \multicolumn{3}{|c|}{ Strahlen $(50 \mu \mathrm{m} / 1$ bar $)$} \\
\hline Befestigung & A & A & SA & A & $(\mathrm{K})$ & SA & A & K & SA \\
\hline $\begin{array}{l}\text { Indikation bei } \\
\text { Bruxismus }\end{array}$ & $(\checkmark)$ & $x$ & $x$ & $x$ & \multicolumn{2}{|l|}{$x$} & \multicolumn{3}{|c|}{$(\checkmark)$} \\
\hline
\end{tabular}

- Tab. 2 Indikationsbereiche einiger zahnfarbener Werkstoffe laut Herstellerangaben.

\begin{tabular}{|l|l|l|l|l|l|l|l|} 
& PAEK & CAD/CAM- & PICN & $\begin{array}{l}\text { Feldspat- } \\
\text { keramiken }\end{array}$ & $\begin{array}{l}\text { leuzitverstärkte } \\
\text { Glaskeramiken }\end{array}$ & $\begin{array}{l}\text { Lithium-X-Silikat- } \\
\text { keramiken }\end{array}$ & $\begin{array}{l}\text { Zirkoniumdioxid- } \\
\text { keramiken (3/4/5Y-TZP) }\end{array}$ \\
\hline Inlays, Onlays & - & + & + & + & + & + & + \\
\hline Veneers & - & + & + & + & + & + & + \\
\hline Kronen FZ & + & + & + & + & + & + & + \\
\hline Kronen SZ & + & + & + & + & + & + & + \\
\hline Brücken & $+^{4}$ & $+^{4}$ & - & - & - & + & + \\
\hline Abutments & $+^{4}$ & - & - & - & - & + & + \\
\hline
\end{tabular}

1 Z. B. PEKK (Polyaryl-Ether-Keton-Keton; Beispiel Pekkton ivory, Cendres + Métaux SA, Biel, Schweiz); ${ }^{2}$ YY-TZP; ${ }^{3}$ zugelassen für 3-gliedrige Endpfeilerbrücken bis 2. PM; gilt für Lithiumdisilikatkeramiken und bedingt für gepresste ZLS; ${ }^{4}$ bitte entsprechende Herstellerangaben berücksichtigen

GmbH, Hamburg, Deutschland; Ambarino high-class, Creamed GmbH \& Co., Marburg, Deutschland). Zudem weisen CAD/CAM-Komposite eine hohe Kantenstabilität auf, weshalb sie im Bereich der Präparationsgrenze dünn auslaufend gestaltet werden können. Dadurch wird ein harmonischer Übergang von natürlichem Zahn und restaurativer Krone ermöglicht. Somit stellen die CAD/ CAM-Komposite auf dem Gebiet der zahnfarbenen Werkstoffe eine Alternative zu keramischen Materialien dar.

\section{Keramiken}

Keramische Restaurationen bilden typischerweise die von Patienten und Behandlern geforderten ästhetischen Eigenschaften bei gleichzeitiger Biokompatibilität und hoher mechanischen Leistungsfähigkeit sehr gut ab und verfügen über ein breites Indikationsspektrum. Bei den Keramiken stellen Silikat- und Oxidkeramiken die beiden
Hauptgruppen dar. Silikatkeramiken bestehen aus Kristallen und Gläsern, deren grundlegender Rohstoff die Silikate $\left(\mathrm{SiO}_{2}\right)$ darstellen [11]. Aus diesem Grund werden in der Zahnmedizin die Begriffe Glas- oder Silikatkeramik häufig synonym verwendet. Im Unterschied zu Kristallen handelt es sich bei Gläsern um unterkühlte Schmelzen, bei denen durch zu schnelles Abkühlen die Atome keine ausreichende Zeit hatten, sich regelmäßig anzuordnen; eine Nahordnung ist zwar vorhanden, aber keine Fernordnung [5]. Da das reine $\mathrm{SiO}_{2}$-Glas aufgrund von zu hoher Viskosität und zu hoher Transparenz als dentale Keramik nicht geeignet ist, werden diese Gläser mittels Netzwerkbildern und -wandlern modifiziert. Das neu modulierte Glas ähnelt nun in seinen Hauptbestandteilen dem natürlichen Feldspat Orthoklas, weshalb dieses als Rohmaterial in der Dentalkeramik Anwendung findet [2,5, 11,12]. Bei diesen klassischen Silikatkeramiken handelt 


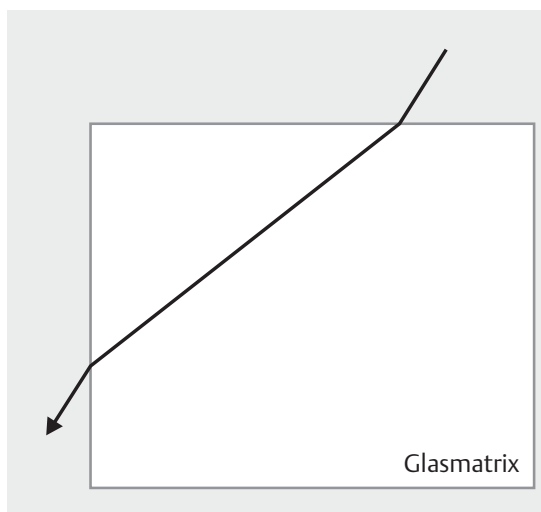

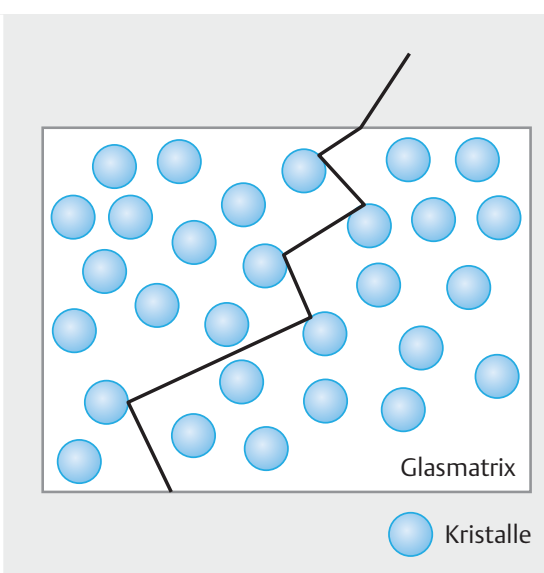

b

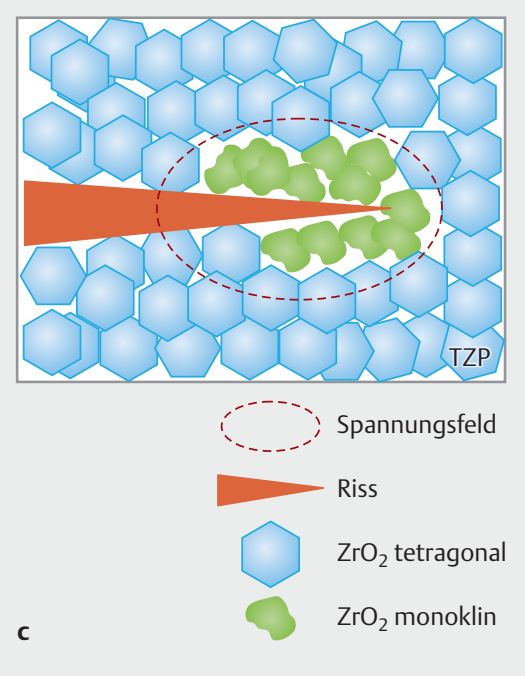

- Abb. 2 Rissverhalten bei verschiedenen Werkstoffen. a Reine Glasmatrix, b Silikatkeramik, c polykristalline tetragonale Zirkoniumdioxidkeramik (TZP).

es sich daher um hochtransluzente Glaskeramiken, die aufgrund ihrer optischen Eigenschaften zahnähnlich wirken und somit optimale ästhetische Ergebnisse liefern. Daher werden sie bevorzugt im Frontzahnbereich als mögliche Versorgungen in Form von Veneers, Teilkronen oder Einzelzahnkronen genutzt [13-15]. Da klassische Silikatkeramiken Biegefestigkeitswerte unter $350 \mathrm{MPa}$ aufweisen, aber für ein konventionelles Zementieren eine Festigkeit von mehr als $350 \mathrm{MPa}$ gefordert ist, wird für Restaurationen aus diesen Werkstoffen in aller Regel eine adhäsive Befestigung notwendig [16].

Die Werkstoffmatrix kann durch Zugabe weiterer Substanzen (z. B. Lithium) optimiert werden, um bspw. höhere Festigkeitswerte zu erreichen oder optische Eigenschaften zu beeinflussen [5]. Hieraus resultieren je nach eingelagertem Kristall Lithium-(X)-silikatkeramiken. Diese weisen im Vergleich zu den klassischen Silikatkeramiken eine höhere Biegefestigkeit und Risszähigkeit auf ( $\vee$ Tab. 1), was in der Konsequenz eine minimalinvasivere Präparation ermöglicht. Kronen aus Lithium-(X)-silikatkeramik können mitunter konventionell befestigt werden. Werkstoffe aus Lithiumdisilikatkeramik und gepresster zirkonverstärkter Lithiumsilikatkeramik (ZLS) werden teilweise auch für die Herstellung von 3-gliedrigen Endpfeilerbrücken im Front- und Prämolarenbereich eingesetzt. In der Implantatprothetik finden sie zudem als Kronen sowie als Hybrid-Abutments Anwendung.

Daneben existieren Oxidkeramiken mit nahezu reinen polykristallinen Festkörpern ohne Glasphase. Diese Oxidkeramiken sind auf Basis von Aluminiumoxid $\left(\mathrm{Al}_{2} \mathrm{O}_{3}\right)$ oder Zirkoniumdioxid $\left(\mathrm{ZrO}_{2}\right)$ erhältlich.
Grundsätzlich handelt es sich bei den Keramiken um spröde Werkstoffe. Treten Mikrorisse speziell an der Oberfläche der Versorgung, im Laufe der Verarbeitungsprozesse, beim Einschleifen auf dem zahnärztlichen Behandlungsstuhl oder beim täglichen Einsatz im Mund durch Überschreitung der kritischen Spannungen, auf ( $\triangleright$ Abb. 2), so kommt es zum plötzlichen Versagen (Bruch) des Werkstoffes. Zirkoniumdioxidkeramiken können das Risswachstum durch eine Phasentransformation und eine damit verbundene Volumenzunahme mitunter verlangsamen und den Defekt teilweise schließen. Aufgrund ihrer guten mechanischen Eigenschaften kommen polykristalline tetragonale Zirkoniumdioxidkeramiken (TZP) häufig als Gerüstmaterialien für Kronen und Brücken zum Einsatz (Beispiel: Katana Zirconia, Kuraray Noritake, Kuraray Europe, Hattersheim, Deutschland). Besonders Keramiken aus Zirkoniumdioxid zeichnen sich durch ihre hohe Biegefestigkeit und Härte aus ( $\bullet$ Tab. 1 ). Die daraus resultierende geringe notwendige minimale Schichtdicke erlaubt eine minimalinvasive Präparation. Zudem weisen diese Werkstoffe eine gute Ästhetik bei akzeptabler Lichtdurchlässigkeit auf und ermöglichen gleichzeitig, verfärbte Zähne zu maskieren [16]. Mit der Einführung der CAD/CAM-Technologie konnten ihre Produktion vereinfacht und die anfallenden Herstellungskosten erheblich gesenkt werden [17]. Auch dem bekannten Problem des „Chippings“ bei verblendeten Restaurationen mit Zirkoniumdioxidkeramik-Gerüsten [18,19] konnte durch Adaptation der Brennparameter und der Etablierung einer anatoformen Gerüstgestaltung weitgehend entgegengewirkt werden [17]. Zudem hat sich die Wahl des Werkstoffes in monolithischer Form, d. h. ohne Verblendung, als Alternative auf dem dentalen Markt etabliert. 
Mittlerweile werden verschiedenste monolithische Restaurationen aus Zirkoniumdioxidkeramik produziert, unter anderem auch ästhetisch ansprechende transluzente Varianten mit 4 Mol.-\% (4Y-TZP) oder 5 Mol.-\% Yttriumoxid (5Y-TZP). Zudem können monolithische Restaurationen minimalinvasiver und somit gleichzeitig zahnhartsubstanzschonender realisiert werden. So bedarf es bei 3Y-TZP Zirkoniumdioxidkeramiken meist einer Reduktion im okklusalen und zirkulären Bereich von ungefähr $0,5 \mathrm{~mm}$. Eine Sonderstellung nehmen die glasinfiltrierten Oxidkeramiken ein, die sowohl polykristalline- als auch silikatkeramische Anteile enthalten. Sie werden den Oxidkeramiken zugeordnet [11], sind aber in den letzten Jahren weitestgehend vom dentalen Markt verschwunden.

Neben diesen beiden Hauptgruppen existieren die Hybridkeramiken bzw. polymerinfiltrierten Keramiken (PICN). Der Begriff Hybridkeramik bezeichnet eine auf dem Dentalmarkt verfügbare Materialgruppe, die eine duale Feldspatkeramik-Polymer-Netzwerkstruktur aufweist (VITA ENAMIC, VITA Zahnfabrik, Bad Säckingen, Deutschland). Das Feldspatkeramiknetzwerk sorgt hierbei für die notwendige Stabilität und Ästhetik, während das Polymernetzwerk die gewünschte Kantenstabilität und die Herstellung auch in dünner Schichtdicke realisiert. Der dentinähnliche Biegemodul ermöglicht, dass Kaukräfte absorbiert werden und Patienten ein zahnähnliches Kaugefühl wahrnehmen. Mit der Einführung von mehrfarbigen Blöcken (VITA ENAMIC multiColor, VITA Zahnfabrik) werden vom Hersteller zudem monolithische Restaurationen aus Hybridkeramik für den ästhetischen Bereich beworben.

\section{Zahnfarbene Werkstoffe und Funktion}

Die Indikationsgebiete von zahnfarbenen Werkstoffen sind aufgrund ihrer ästhetischen (Transluzenz, Farbe, Opazität, Reflexion) und mechanischen Eigenschaften (Biegefestigkeit, Härte und E-Modul) verschieden definiert. Zudem wird versucht, Patientenwünsche zu berücksichtigen. Zusätzlich sollten Parameter wie die notwendige Schichtdicke, die materialabhängige Art der Befestigung sowie die Art der Fertigung für den langfristigen Therapieerfolg berücksichtigt werden. Speziell bei Patienten mit Parafunktionen, im Besonderen Bruxismus, stellt die Therapie eine große Herausforderung in der täglichen Praxis dar.

\section{Indikation und Limitation bei Patienten mit Bruxismus}

Bruxismus ist definiert als „eine wiederholte Kaumuskelaktivität, charakterisiert durch Kieferpressen und Zähneknirschen und/oder Anspannen oder Verschieben des Unterkiefers ohne Zahnkontakt“ [20]. Im Detail können 2 zirkadiane Erscheinungsformen von Bruxismus unterschieden werden - Schlafbruxismus (SB) und Wachbruxismus (WB) [21]. Dabei ist Schlafbruxismus eher als zen- tralnervöse Störung zu sehen, während Wachbruxismus mit psychologischen Faktoren wie emotionalem Stress in Zusammenhang gebracht wird [20,22,23]. Grundsätzlich ist die prothetische Versorgung von Patienten mit Bruxismus immer mit besonderer Aufmerksamkeit anzufertigen. Bei implantatprothetischen Versorgungen wird teilweise ein stark erhöhtes Risiko (3,4-fach) für Implantatfrakturen bei Patienten mit Bruxismus angegeben [24]. Bei implantatprothetischen Arbeiten sollten daher eher konservative Ansätze gewählt und Komplikationen wie Lockerungen von Implantatschrauben oder starke Abnutzungen der Okklusionsflächen gründlich kontrolliert werden. Für die Performance von zahngetragenen prothetischen Restaurationen bleibt der Einfluss von Bruxismus weiterhin nicht abschließend geklärt. Auch die Frage, ob Patienten mit Bruxismus mit vollkeramischen Versorgungen erfolgreich therapiert werden können, kann laut aktueller S3-Leitlinie zu vollkeramischen Kronen und Brücken nicht abschließend bewertet werden [33]. Dies ist besonders darin begründet, dass in nur sehr wenigen Studien zu vollkeramischen Versorgungen Patienten mit bekanntem Bruxismus eingeschlossen wurden [25]. Zudem erhöht die Diagnostik von Bruxismus die Heterogenität der Studienergebnisse, da er häufig durch verschiedene Methoden ermittelt wird. Momentan kann nur die Polysomnografie einen Bruxismus definitiv bestätigen; dieses Verfahren ist jedoch zeit- und kostenintensiv und wird daher in Studien nur selten genutzt. Im Sinne der Aktualisierung der S3-Leitlinie zu Vollkeramik bleiben aktuelle Empfehlungen zur Versorgung bei Patienten mit Bruxismus abzuwarten.

Falls eine zahnfarbene Restauration aus Keramik bei Patienten mit Bruxismus in Erwägung gezogen wird, sollte eine monolithische Anwendung präferiert werden, da ein Chipping häufiger bei parafunktionellen Aktivitäten auftreten kann und im Zusammenhang mit Vollkeramiken vermehrt beschrieben wird [26,27]. Frühere Annahmen, Restaurationen aus hochfesten Keramiken (z. B. Zirkoniumdioxidkeramik) könnten zu einer erhöhten Abrasion der Antagonisten führen, konnten von verschiedenen wissenschaftlichen Forschungsgruppen widerlegt werden. So zeigten Studienergebnisse, dass sorgsam polierte Zirkoniumdioxidkeramik-Oberflächen eine geringere Abrasion der Antagonisten im Vergleich zu klassischen Verblendkeramiken oder auch Lithiumdisilikatkeramiken verursachten [28-32]. Nichtsdestotrotz kann die monolithische Anwendung von Zirkoniumdioxidkeramiken ästhetisch limitierend sein, wenngleich kleinere Individualisierungen durch das Bemalen der Keramik möglich sind. Daher muss bspw. bei ausgefalleneren Zahnfarben oder transluzenten Bereichen auf eine Verblendung im ästhetisch relevanten Bereich mit bspw. Dentin- oder Schmelzmassen zurückgegriffen werden ( $\bullet$ Abb. 3 ). 

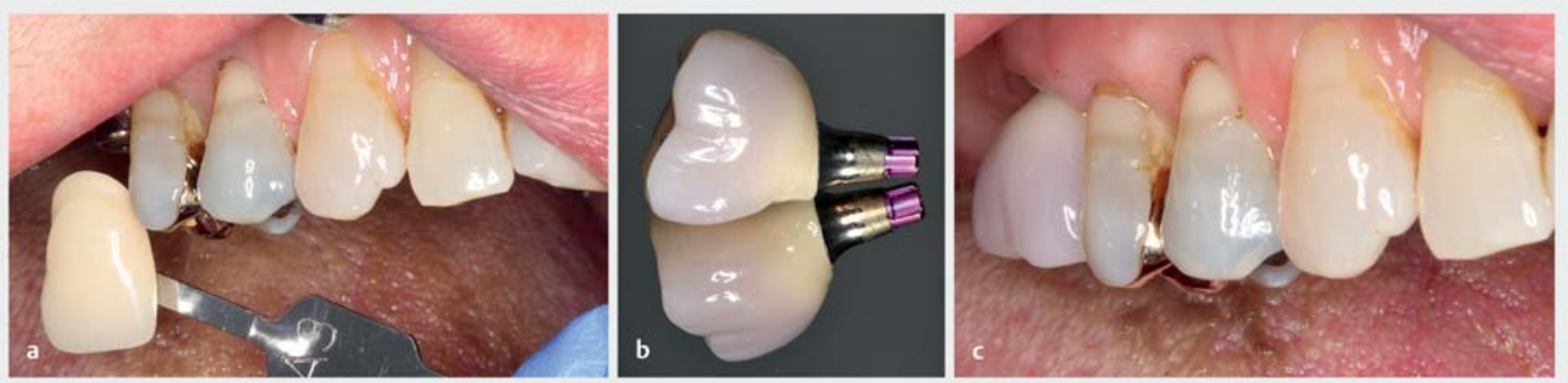

- Abb. 3 a Die Bestimmung der Zahnfarbe ist aufgrund der bläulichen Schmelzanteile nicht eindeutig; anstelle der monolithischen Anwendung wird die ... b Lithiumdisilikatkeramikkrone mittels Schichttechnik vestibulär verblendet und fügt sich ... c ästhetisch ins Gesamtbild ein.

Gemäß den Herstellerangaben sind momentan keine Silikatkeramiken/Hybridkeramiken auf dem dentalen Markt verfügbar, die für Bruxismus indiziert sind. Wenige Zirkoniumdioxidkeramiken sind seit einiger Zeit bei Parafunktionen für bestimmte Indikationen zugelassen bzw. nicht kontraindiziert (BruxZir Solid Zirconia, Newport Beach, CA, USA; LAVA Zirkonoxid, 3M; VITA YZ HT, Vita Zahnfabrik). Besonders interessant sind auch die CAD/CAMKomposite, die seitens der Hersteller teilweise sogar für Patienten mit Bruxismus beworben werden (LuxaCam Composite, DMG, Hamburg, Deutschland). Nach Anfertigung des Zahnersatzes sollte eine Schiene in Erwägung gezogen werden, welche die Restauration vor den parafunktionellen Belastungen schützt [27].

\section{Langzeitbeobachtungen und Überlebensdaten}

Da nahezu keine Studien zu zahnfarbenen Versorgungen bei Patienten mit Parafunktionen vorliegen, wird nachfolgend auf grundsätzliche Langzeitbeobachtungen und Überlebensdaten zahnfarbener Restaurationen eingegangen. In der aktuellen S3-Leitlinie zu vollkeramischen Restaurationen wurden evidenzgestützte Ergebnisse zur klinischen Bewährung vollkeramischer Restaurationen analysiert und veröffentlicht [33]. In dieser wird für die Herstellung von Front- und Seitenzahnkronen die monolithische Anwendung von Lithiumdisilikat- gegenüber der verblendeten Lithiumdisilikatkeramik vorgezogen (Vermeidung eines Chippings). Dennoch belegen Studien, dass die Überlebensrate von Restaurationen aus verblendeter Lithiumsilikatkeramik nach 8 Jahren bei $94,8 \%$ liegt [34]. Ihre monolithischen und im Chairside-Verfahren gefertigten Pendants erreichten eine Überlebensrate von $83,5 \%$ nach 10 Jahren [35]. Gemeinhin zeigte sich, dass die Überlebensraten von vollkeramischen Front- sowie Seitenzahnkronen vergleichbar mit jenen aus Metallkeramik sind [36-38].
Eine Studie, die das Verhalten von verblendeten Zirkoniumdioxidkeramiken anhand von 137 Einzelzahnkronen evaluierte, belegte, dass das Gesamtüberleben nach einem Beobachtungszeitraum von 5 Jahren bei 94,7\% lag. Komplikationen wie Haarrisse oder keramische Abplatzungen wurden häufiger bei Patienten mit Parafunktionen beobachtet als bei funktionell unauffälligen Patienten [39]. Aufgrund der zufriedenstellenden positiven Datenlage und zufriedenstellenden Überlebensraten nach bis zu 6 Jahren Beobachtungszeit können für 3-gliedrige Brücken im Frontzahnbereich Restaurationen aus Zirkoniumdioxidkeramik empfohlen werden $[40,41]$. Gemeinhin zeigten sich in Studien zu 3-gliedrigen Seitenzahnbrücken mit einem Gerüst aus Zirkoniumdioxidkeramik innerhalb eines Beobachtungszeitraums von mind. 5 Jahren Überlebensraten von 90,0\% [42-48]. Die Komplikationen im Sinne von Chipping waren jedoch teilweise sehr hoch $[49,50]$. Zu vollkeramischen 2- oder mehrspannigen Brücken liegt gemäß S3-Leitlinie keine ausreichende wissenschaftliche Evidenz vor [33].

Restaurationen (Einzelkronen) aus CAD/CAM-Kompositen zeigten in ersten klinischen Studien Überlebensraten von 85,7\% nach 24 Monaten [51]. Zum jetzigen Zeitpunkt liegen allerdings noch keine Daten zur Langzeitbewährung vor, weshalb Studien mit längeren Beobachtungszeiträumen abzuwarten sind, bevor weitere Aussagen zu Überlebens- und Erfolgsraten von CAD/CAMKompositen getroffen werden können.

\section{Zusammenfassung}

Nach heutigem Stand sind der Verwendung von zahnfarbenen Werkstoffen kaum Grenzen gesetzt. Somit stehen dem behandelnden Zahnarzt in der Praxis neben den mittlerweile sehr weit verbreiteten Silikat- und Zirkoniumdioxidkeramiken auch CAD/CAM-Komposite als mögliche Therapieoptionen zur Verfügung. Um in Abhängigkeit von der individuellen Patientensituation das adäquate Material auszuwählen, sollte der Behandler über die jeweiligen mechanischen sowie optischen Eigenschaf- 
ten, die Art der Fertigung und die materialabhängigen Befestigungsvoraussetzungen informiert sein. Im Allgemeinen haben sich dentale Keramiken und indirekte Komposite in klinischen Studien bewährt. Es fehlen jedoch aussagekräftige Studien, die Langzeitbeobachtungen zahnfarbener Werkstoffe bei Patienten mit funktionsdiagnostischen Auffälligkeiten einschließen. Besonders das Vorhandensein eines Bruxismus ist eine Kontraindikation für die Anwendung vieler zahnfarbener Materialien und sollte individuell mit den Informationen der Hersteller in regelmäßigen zeitlichen Abständen verglichen werden. Einige CAD/CAM-Komposite und Zirkoniumdioxidkeramiken sind mittlerweile für zahlreiche prothetische Restaurationsformen bei Bruxismus zugelassen und können als Therapiealternative zu metall(keram) ischen Restaurationen erwogen werden.

\section{Interessenkonflikt}

Die Autorinnen/Autoren geben an, dass kein Interessenkonflikt besteht.

\section{Autorinnen/Autoren}

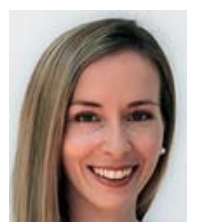

Dr. med. dent. Anuschka Josephine Roesner

Poliklinik für Zahnärztliche Prothetik und Werkstoffkunde, Universität Leipzig

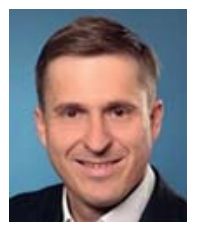

Dr. rer. nat. Dipl.-Ing. Andreas König Poliklinik für Zahnärztliche Prothetik und Werkstoffkunde, Universität Leipzig

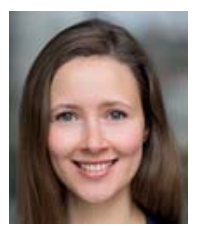

\section{Zahnärztin Elena Günther}

Poliklinik für Zahnärztliche Prothetik und Werkstoffkunde, Universität Leipzig

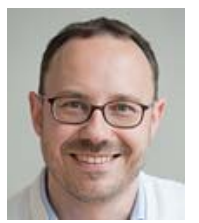

Prof. Dr. med. dent. Sebastian Hahnel

Poliklinik für Zahnärztliche Prothetik und Werkstoffkunde, Universität Leipzig

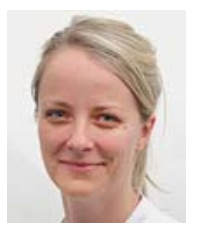

Poliklinik für Zahnärztliche Prothetik und Werkstoffkunde, Universität Leipzig
Korrespondenzadresse

\section{Dr. Anuschka Roesner}

Poliklinik für Zahnärztliche Prothetik und Werkstoffkunde Universitätsklinikum Leipzig

04103 Leipzig

Deutschland

anuschka.roesner@medizin.uni-leipzig.de

\section{Literatur}

[1] Li RWK, Chow TW, Matinlinna JP. Ceramic dental biomaterials and CAD/CAM technology: state of the art. J Prosthodont Res 2014; 58: 208-216

[2] Guess PC, Schultheis S, Bonfante EA et al. All-ceramic systems: laboratory and clinical performance. Dent Clin North Am 2011; 55: 333-352

[3] Baumgartner S, Gmeiner R, Schönherr JA et al. Stereolithography-based additive manufacturing of lithium disilicate glass ceramic for dental applications. Materials Science and Engineering: C 2020. doi:10.1016/j.msec.2020.111180

[4] Methani MM, Revilla-León M, Zandinejad A. The potential of additive manufacturing technologies and their processing parameters for the fabrication of all-ceramic crowns: A review. J Esthet Restor Dent 2020; 32: 182-192

[5] Behr M, Belli R, Boccaccini AR, Forstreuter J, Frankenberger R, Gbureck U, Gmeiner R, Hahnel S, Ilie N, Kraft M, Lohbauer U, Mehl A, Preis V, Reich S, Rosentritt M, Schmidtke M, Snétivy D, Stampfl J, Stawarczyk B, Strietzel R, Wendler M, Will J, Wöstmann B, Zimmermann M. Werkstoffkunde in der Zahnmedizin: Moderne Materialien und Technologien. Stuttgart, New York: Thieme; 2018

[6] Stawarczyk B, Beuer F, Wimmer T et al. Polyetheretherketonea suitable material for fixed dental prostheses? J Biomed Mater Res Part B Appl Biomate 2013; 101: 1209-1216

[7] Fokas G, Guo CY, Tsoi JKH. The effects of surface treatments on tensile bond strength of polyether-ketone-ketone (PEKK) to veneering resin. J Mech Behav Biomed Mater 2019; 93: 1-8

[8] Burke FJT, Mackenzie L, Shortall ACC. Survival rates of resin composite restorations in loadbearing situations in posterior teeth. Dent Update 2019; 46: 524-536

[9] Chavali R, Nejat AH, Lawson NC. Machinability of CAD-CAM materials. J Prosthet Dent 2017; 118: 194-199

[10] Rauch A, König A. Indirekte Komposite aus klinischer und werkstoffkundlicher Sicht. Quintessenz 2020; 71: 116-126

[11] Pospiech P, Tinschert J, Raigrodski A. Keramik-Vollkeramik Kompendium. Seefeld: 3M Espe; 2005. Im Internet (Stand 24.11.2020): https://multimedia.3m.com/mws/media/ 5987970/lava-keramik-vollkeramik-kompendium.pdf?fn= Lava_Vollkeramik_Kompend_D.pdf

[12] Edelhoff D. Vollkeramik von A bis Z für Praktiker. ZWR - Das Deutsche Zahnärzteblatt 2003; 112: 276-281

[13] Magne P, Douglas WH. Additive contour of porcelain veneers: a key element in enamel preservation, adhesion, and esthetics for aging dentition. J Adhes Dent 1999; 1: 81-92

[14] van Dijken, Jan W V, Hasselrot L. A prospective 15-year evaluation of extensive dentin-enamel-bonded pressed ceramic coverages. Dent Mater 2010; 26: 929-939
[15] Frankenberger R, Taschner M, Garcia-Godoy F et al. Leucite-reinforced glass ceramic inlays and onlays after 12 years. J Adhes Dent 2008; 10: 393-398 
[16] Kern M, Beuer F, Frankenberger R, Kohal RJ, Kunzelmann KH, Mehl A, Pospiech P, Reiss B. Vollkeramik auf einen Blick: Leitfaden zur Indikation, Werkstoffauswahl, Vorbereitung und Eingliederung von vollkeramischen Restaurationen. 6. dt., erw. Aufl. Ettlingen: AG Keramik; 2015

[17] Nam J, Tokutomi H. Using zirconia-based prosthesis in a complete-mouth reconstruction treatment for worn dentition with the altered vertical dimension of occlusion. J Prosthet Dent 2015; 113: 81-85

[18] Lima E de, Meira JBC, Özcan M et al. Chipping of Veneering Ceramics in Zirconium Dioxide Fixed Dental Prosthesis. Curr Oral Health Rep 2015; 2: 169-117

[19] Swain MV. Unstable cracking (chipping) of veneering porcelain on all-ceramic dental crowns and fixed partial dentures. Acta Biomater 2009; 5: 1668-1677

[20] Peroz I, Bernhardt O, Kares H et al. Diagnostik und Behandlung von Bruxismus: S3-Leitlinie. Im Internet (Stand: 21.07.2020): www.awmf.org/uploads/tx_szleitlinien/083-027l_S3_Bruxismus-Diagnostik-Behandlung_2019-06.pdf

[21] Lobbezoo F, Ahlberg J, Raphael KG et al. International consensus on the assessment of bruxism: Report of a work in progress. J Oral Rehabil 2018; 45: 837-844

[22] Castrillon EE, Ou K-L, Wang K et al. Sleep bruxism: an updated review of an old problem. Acta Odontol Scand 2016; 74: 328334

[23] Shetty S, Pitti V, Satish Babu CL et al. Bruxism: a literature review. J Indian Prosthodont Soc 2010; 10: 141-148

[24] Rauch A, Schmidt M, Schierz O. Wie viel Risiko - wie viel Sicherheit? Implantation bei Patienten mit Bruxismus. ZZI 2019; 35: $284-289$

[25] Melo G, Duarte J, Pauletto P et al. Bruxism: An umbrella review of systematic reviews. J Oral Rehabil 2019; 46: 666-690

[26] Karl M. Outcome of bonded vs. all-ceramic and metal-ceramic fixed prostheses for single tooth replacement. Eur J Oral Implantol 2016; 9 (Suppl 1): S25-S44

[27] Koenig V, Vanheusden AJ, Le Goff SO et al. Clinical risk factors related to failures with zirconia-based restorations: an up to 9-year retrospective study. J Dent 2013; 41: 1164-1174

[28] Jung Y-S, Lee J-W, Choi Y-J et al. A study on the in-vitro wear of the natural tooth structure by opposing zirconia or dental porcelain. J Adv Prosthodont 2010; 2: 111-115

[29] Rosentritt M, Preis V, Behr M et al. Two-body wear of dental porcelain and substructure oxide ceramics. Clin Oral Investig 2012; 16: 935-943

[30] Preis V, Behr M, Kolbeck C et al. Wear performance of substructure ceramics and veneering porcelains. Dent Mater 2011; 27: 796-804

[31] Kontonasaki E, Rigos AE, Ilia C et al. Monolithic Zirconia: An Update to Current Knowledge. Optical Properties, Wear, and Clinical Performance. Dent J (Basel) 2019; 7: 90

[32] Pathan MS, Kheur MG, Patankar AH et al. Assessment of Antagonist Enamel Wear and Clinical Performance of Full-Contour Monolithic Zirconia Crowns: One-Year Results of a Prospective Study. J Prosthodont 2018; 28: e411-e416

[33] Meyer G, Ahsbahs S, Kern M. Vollkeramische Kronen und Brücken: S3-Leitlinie (31.08.2014). Im Internet (Stand: 21.07.2020): www.awmf.org/uploads/tx_szleitlinien/083012l_S3_Vollkeramische_Kronen_Br\%C3\%BCcken_2015-04abgelaufen.pdf

[34] Gehrt M, Wolfart S, Rafai N et al. Clinical results of lithium-disilicate crowns after up to 9 years of service. Clin Oral Investig 2013; 17: 275-284
[35] Rauch A, Reich S, Dalchau L et al. Clinical survival of chair-side generated monolithic lithium disilicate crowns:10-year results. Clin Oral Investig 2018; 22: 1763-1769

[36] Kerschbaum T. Langzeitüberlebensdauer von Zahnersatz: eine Übersicht. Quintessenz 2004; 55: 1113-1126

[37] Walton TR. A 10-year longitudinal study of fixed prosthodontics: clinical characteristics and outcome of single-unit metalceramic crowns. Int J Prosthodont 1999; 12: 519-526

[38] Walton TR. The up to 25-year survival and clinical performance of 2,340 high gold-based metal-ceramic single crowns. Int J Prosthodont 2013; 26: 151-160

[39] Monaco C, Caldari M, Scotti R. Clinical evaluation of 1,132 zirconia-based single crowns: a retrospective cohort study from the AIOP clinical research group. Int J Prosthodont 2013; 26: 435-442

[40] Lops D, Mosca D, Casentini P et al. Prognosis of zirconia ceramic fixed partial dentures: a 7-year prospective study. Int J Prosthodont 2012; 25: 21-23

[41] Molin MK, Karlsson SL. Five-year clinical prospective evaluation of zirconia-based Denzir 3-unit FPDs. Int ] Prosthodont 2008; 21: $223-227$

[42] Kern M, Sasse M, Wolfart S. Ten-year outcome of three-unit fixed dental prostheses made from monolithic lithium disilicate ceramic. J Am Dent Assoc 201; 143: 234-240

[43] Makarouna M, Ullmann K, Lazarek K et al. Six-year clinical performance of lithium disilicate fixed partial dentures. Int J Prosthodont 2011; 24: 204-206

[44] Pjetursson BE, Lang NP. Prosthetic treatment planning on the basis of scientific evidence. J Oral Rehabil 2008; 35 (Suppl 1): 72-79

[45] Chun Y-HP, Raffelt C, Pfeiffer $\mathrm{H}$ et al. Restoring strength of incisors with veneers and full ceramic crowns. J Adhes Dent 2010; 12: 45-54

[46] Eschbach S, Wolfart S, Bohlsen F et al. Clinical evaluation of allceramic posterior three-unit FDPs made of In-Ceram Zirconia. Int J Prosthodont 2009; 22: 490-492

[47] Sorrentino R, Simone G de, Tetè S et al. Five-year prospective clinical study of posterior three-unit zirconia-based fixed dental prostheses. Clin Oral Investig 2012; 16: 977-985

[48] Raigrodski AJ, Hillstead MB, Meng GK et al. Survival and complications of zirconia-based fixed dental prostheses: A systematic review. J Prosthet Dent 2012; 107: 170-177

[49] Sailer I, Strasding M, Valente NA et al. A systematic review of the survival and complication rates of zirconia-ceramic and metal-ceramic multiple-unit fixed dental prostheses. Clin Oral Implants Res 2018; 29 (Suppl 16): 184-198

[50] Morton D, Gallucci G, Lin W-S et al. Group 2 ITI Consensus Report: Prosthodontics and implant dentistry. Clin Oral Implants Res 2018; 29 (Suppl 16): 215-223

[51] Zimmermann M, Koller C, Reymus M et al. Clinical Evaluation of Indirect Particle-Filled Composite Resin CAD/CAM Partial Crowns after 24 Months. J Prosthodont 2018; 27: 694-699

\section{Bibliografie}

ZWR - Das Deutsche Zahnärzteblatt 2020; 129: 626-633

DOI 10.1055/a-1300-0205

ISSN 0044-166X

C 2020. Thieme. All rights reserved.

Georg Thieme Verlag KG, Rüdigerstraße 14,

70469 Stuttgart, Germany 